OPEN ACCESS

Edited by:

Vicent Arbona

Jaume I University, Spain

Reviewed by:

Barbara Baldan,

University of Padova, Italy Walter Chitarra,

Consiglio per la Ricerca in Agricoltura e l'Analisi dell'Economia Agraria, Italy

*Correspondence:

Caiqiu Gao

gaocaiqiu@yahoo.com

Specialty section: This article was submitted to

Plant Abiotic Stress,

a section of the journa

Frontiers in Plant Science

Received: 12 November 2016 Accepted: 23 March 2017

Published: 07 April 2017

Citation:

Yang G, Yu L, Wang Y, Wang C and

Gao C (2017) The Translation Initiation Factor $1 A$ (ThelF1A) from Tamarix hispida Is Regulated by a Dof Transcription Factor and Increased Abiotic Stress Tolerance. Front. Plant Sci. 8:513. doi: 10.3389/fpls.2017.00513

\section{The Translation Initiation Factor 1A (ThelF1A) from Tamarix hispida Is Regulated by a Dof Transcription Factor and Increased Abiotic Stress Tolerance}

\author{
Guiyan Yang, Lili Yu, Yucheng Wang, Chao Wang and Caiqiu Gao* \\ State Key Laboratory of Tree Genetics and Breeding, Northeast Forestry University, Harbin, China
}

Eukaryotic translation initiation factor $1 \mathrm{~A}$ (elF1A) functions as an mRNA scanner and AUG initiation codon locator. However, few studies have clarified the role of elF1A in abiotic stress. In this study, we cloned elF1A (ThelF1A) from Tamarix hispida and found its expression to be induced by $\mathrm{NaCl}$ and polyethylene glycol (PEG) in roots, stems, and leaves. Compared to control, ThelF1A root expression was increased 187.63fold when exposed to $\mathrm{NaCl}$ for $6 \mathrm{~h}$, suggesting a potential abiotic stress response for this gene. Furthermore, transgenic tobacco plants overexpressing ThelF1A exhibited enhanced seed germination and a higher total chlorophyll content under salt and mannitol stresses. Increased superoxide dismutase, peroxidase, glutathione transferase and glutathione peroxidase activities, as well as decreased electrolyte leakage rates and malondialdehyde contents, were observed in ThelF1A-transgenic tobacco and T. hispida seedlings under salt and mannitol stresses. Histochemical staining suggested that ThelF1A improves reactive oxygen species (ROS) scavenging in plants. Moreover, ThelF1A may regulate expression of stress-related genes, including TOBLTP, GST, MnSOD, NtMPK9, poxN1, and CDPK15. Moreover, a 1352-bp promoter fragment of ThelF1A was isolated, and cis-elements were identified. Yeast one-hybrid assays showed that ThDof can specifically bind to the Dof motif present in the promoter. In addition, ThDof showed expression patterns similar to those of ThelF1A under $\mathrm{NaCl}$ and PEG stresses. These findings suggest the potential mechanism and physiological roles of ThelF1A. ThDof may be an upstream regulator of ThelF1A, and ThelF1A may function as a stress response regulator to improve plant salt and osmotic stress tolerance via regulation of associated enzymes and ROS scavenging, thereby reducing cell damage under stress conditions.

Keywords: elF1A, expression, abiotic stress, promoter, Tamarix hispida

Abbreviations: $\mathrm{ABA}$, abscisic acid; $\mathrm{COR}$, cold responsive; $\mathrm{DAB}, 3,3^{\prime}$-diaminobenzidine tetrahydrochloride; EL, electrolyte leakage; GPX, glutathione peroxidase; GST, glutathione transferase; $\mathrm{H}_{2} \mathrm{DCF}-\mathrm{DA}, 2$, 7-dichlorofluorescein diacetate; MDA, malondialdehyde; MW, molecular weight; pI, isoelectric point; POD, peroxidase; qRT-PCR, quantitative real-time polymerase chain reaction; ROS, reactive oxygen species; SOD, superoxide dismutase; tcc, total chlorophyll content. 


\section{INTRODUCTION}

Eukaryotic initiation factors (eIFs), including six different families with various functions, namely, eIF1, eIF2, eIF3, eIF4, eIF5 and eIF6, are involved in translation initiation in eukaryotes (Howarth, 1991; Pyronnet and Sonenberg, 2001; Dong and Zhang, 2006). In addition, eIFs are involved in aspects of stress regulation. For example, strong heat shock in Saccharomyces cerevisiae induces assembly of stress granules containing eIF3 that is independent of eIF2 $\alpha$ phosphorylation (Groušl et al., 2009). TaeIF3 $g$ is expressed in response to mild osmotic stress, an effect that differed in the grains of two wheat cultivars (Singh et al., 2007). eIF4E isoform 2 is a salt-related factor in Schizosaccharomyces pombe (Ptushkina et al., 2004), whereas eIF5A was initially identified as a factor involved in the formation of the first peptide bond in rabbit reticulocytes (Li et al., 2004; Costa-Neto et al., 2006). eIF5A was subsequently identified as a factor important for plant adaptation to changing environmental conditions by increasing protein synthesis and enhancing ROS scavenging. This latter aspect is related to increased SOD and POD activities as well as the prevention of chlorophyll loss and membrane damage (Wang et al., 2012).

The translation initiation factor $e I F 1 A$ is necessary for directing the $43 \mathrm{~S}$ preinitiation complex from the $5^{\prime}$ end of an mRNA to the initiation codon in a process referred to as 'scanning.' In humans, eIF1A contains an oligonucleotidebinding (OB) fold and binds to single-stranded RNA oligonucleotides in a site-specific but non-sequence-specific manner, suggesting mRNA interaction rather than specific rRNA or tRNA binding (Battiste et al., 2000). Translation initiation proceeds via an initiator tRNA and the start codon of an mRNA positioned in the ribosomal $\mathrm{P}$ site. In eukaryotes, one of the first steps involves the binding of $e I F 1$ and $e I F 1 A$ to the 40 S ribosomal subunit $e I F 1$ and the $\operatorname{IF} 1 A$ promoter in an open, scanningcompetent preinitiation complex that closes following start codon recognition (Passmore et al., 2007). Previous studies have demonstrated that IFIA participates in plant developmental and stress regulation. For instance, overexpression of sugar beet eIF1A specifically increased sodium and lithium salt tolerance in yeast and Arabidopsis, which suggests that BveIF1A is an important determinant of sodium tolerance (Rausell et al., 2003). Expression of OseIF1 in Oryza sativa was up-regulated treatment with salt, ABA and mannitol; moreover, OseIF1 plays a central role in salt stress adaptation in rice by regulating ion accumulation and the intracellular redox status (Diédhiou et al., 2008; Rangan et al., 2009). eIF1 expression in Porteresia coarctata was also found to be induced by $\mathrm{NaCl}, \mathrm{ABA}$, and mannitol treatments (Latha et al., 2004). Regardless, few studies have focused on IF1A-mediated abiotic stress regulation in woody plants. Accordingly, further investigation is needed to determine how physiological changes are controlled through eIF1A, such as the promotion of ROS scavenging systems to adjust to various environments.

Tamarix hispida, a shrub or small tree primarily distributed in saline or arid and semi-arid regions, is highly tolerant to salt, drought, and high temperature. Recently, a novel obligately halophilic, facultatively alkaliphilic actinobacterium, designated
EGI 80759T, was isolated from the rhizosphere of T. hispida Willd, in Karamay, Xinjiang Province, northwestern China. Strain EGI 80759T exhibits obligately halophilic growth with tolerance to $8-25 \%(\mathrm{w} / \mathrm{v}) \mathrm{NaCl}$ (optimum growth at $10-12 \%$, $\mathrm{w} / \mathrm{v}$ ) and facultatively alkaliphilic growth within the $\mathrm{pH}$ range 7.0-11.0 (optimum growth at pH 9.0-10.0) (Zhang et al., 2016). Based on monitoring data of the groundwater level at typical sections in the lower reaches of Tarim River, the PSII photosynthetic activity of $T$. hispida under drought stress appears to decrease with increasing groundwater depth, and the increase in excess energy could result in a greater risk of photoinhibition. However, the good adaptability and drought tolerance of $T$. hispida could prevent serious PSII damage despite the presence of drought stress (Zhu et al., 2010). In addition, some abiotic stress response genes of $T$. hispida have been characterized, such as the V-type $\mathrm{H}^{+}$-ATPase $\mathrm{c}$ subunit (ThVHAc1) (Yang et al., 2016), WRKY transcript factor (Zheng et al., 2013), and GST (Yang et al., 2014). Such information makes T. hispida an ideal model for cloning stress tolerance genes and investigating the physiological and molecular mechanisms of stress responses in trees (Gao et al., 2008). In the current study, we cloned and characterized the eIF1A gene from $T$. hispida and found it to be strongly responsive to salt and osmotic stresses. TheIF1A improved salt and osmotic stress tolerance in transgenic plants by regulating stress-related gene expression, improving plant ROS scavenging ability, and decreasing cell damage and death. The results of these experiments provide evidence regarding the potential mechanism and physiological role of eIF1A in T. hispida.

\section{RESULTS}

\section{Cloning and Analysis of ThelF1A and its Promoter}

The full-length TheIF1A gene is 432 bp and encodes a 143aa protein with a predicted molecular weight of $16.40 \mathrm{kDa}$ and a theoretical pI of 5.09. Multiple sequence alignment analysis indicated that the eIF1A proteins from different plants share a highly conserved eIF1A domain (Supplementary Figure S1A) located between residues 28 and 110 but without SANT, TUDOR, FN1, CNX, or PLDc features. Phylogenetic analysis indicates that TheIF1A is most similar to eIF1A from $B$. vulgaris (Supplementary Figure S1B).

A 1352-bp (from -1 to -1352 ) promoter region was cloned using thermal asymmetric interlaced polymerase chain reaction (TAIL-PCR). Searches using the PLACE algorithm demonstrated the presence of many cis-elements, such as ABRELATERD1, CAATBOX1, DOFCOREZM, MYB1AT, TATABOX2, and WRKY71OS, in this region, which indicates that TheIF1A may be regulated by different types of transcription factors (TFs) (Supplementary Figure S2).

\section{Expression of TheIF1A in T. hispida}

The results of qRT-PCR showed TheIF1A to be induced in response to $\mathrm{NaCl}$ and polyethylene glycol (PEG) treatments. 


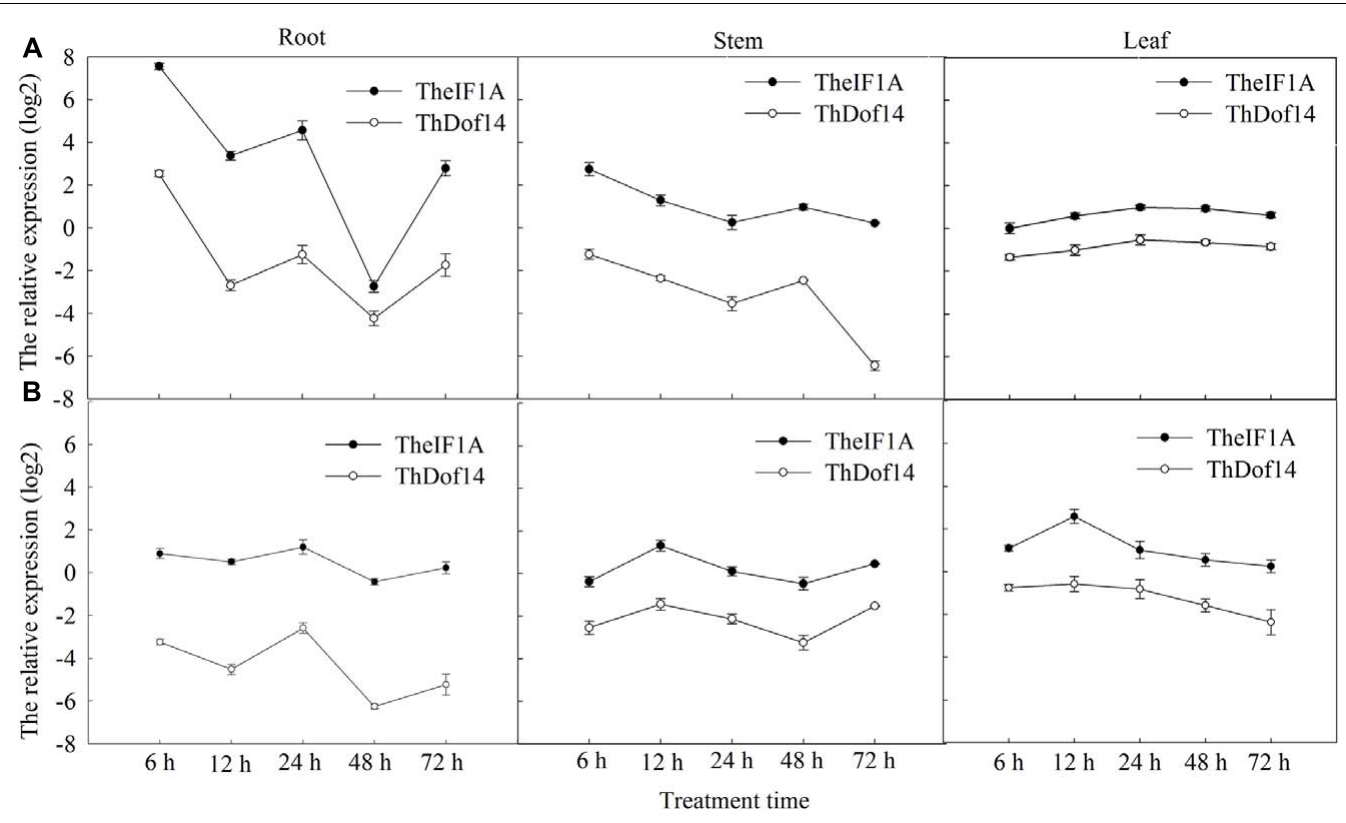

FIGURE 1 | TheIF1A and ThDof expression patterns in different organs of Tamarix hispida in response to various treatments. The relative expression level $=$ transcription level under stress treatment/transcription level under control condition ( 0 h). And all the expression leves were log2 transformed. $\beta$-Actin (FJ618517), $\alpha$-tubulin (FJ618518), and $\beta$-tubulin (FJ618519) were used as reference genes. Error bars were obtained from three replicates of real-time PCR, and every replicate included at least 20 seedlings used as biological replicates. (A) $0.4 \mathrm{M} \mathrm{NaCl}$ stress; (B) 20\% (W/v) PEG6000 stress.

After $\mathrm{NaCl}$ treatment for $6 \mathrm{~h}$, expression of TheIF1A in roots was increased by 187.6-fold compared to the control. At $48 \mathrm{~h}$, TheIF1A expression was down-regulated in roots compared to the control but up-regulated by 1.97-fold in stems (Figure 1A). Under PEG stress, TheIF1A transcription was considerably higher in leaves than in roots and stems, with TheIF1A transcripts in leaves being 4.22-fold higher in roots and 2.43-fold higher in stems at $12 \mathrm{~h}$ (Figure 1B).

To further investigate expression via the TheIF1A promoter, transgenic Arabidopsis plants expressing GUS under the control of the TheIF1A promoter (namely, pTheIF1A::GUS) were generated (Figure 2A). The results showed GUS activity throughout the entire plant, except for in immature seeds of the silique (Figures 2B-P). Furthermore, expression levels differed among tissues and developmental stages. pTheIF1A::GUS was also transiently transformed into $T$. hispida seedlings followed by GUS staining, with the highest GUS activity in roots and the lowest in stems (Figure 2Q-S), further confirming the results for the tissue expression profile of TheIF1A.

\section{Analysis of Upstream Regulators of ThelF1A}

Twelve Dof motifs were identified in the TheIF1A promoter, suggesting that TheIF1A is likely regulated by TFs that interact with this motif. A yeast one-hybrid assay was conducted to identify upstream regulators using the reporter vector pHIS2-cis (containing triple tandem Dof motif repeats) as bait to screen a Tamarix TF cDNA library. One protein (ThDof, KF896302) was found to bind to the Dof motif but failed to bind to mutant Dof motifs (Figure 3A). In addition, ThDof could bind to a truncated TheIF1A promoter retaining the Dof motif but could not bind to promoter fragments lacking the motif or with a mutated Dof motif (Figure 3A). These findings suggest that ThDof may specifically bind to the Dof motif and may regulate TheIF1A by binding to this motif in the TheIF1A promoter.

To validate these interactions, we co-transformed reporter plasmids (the motifs, promoter fragments and serially mutated motifs followed by a 46-bp minimal promoter were independently cloned into pCAMBIA1301) and the effector construct (pROKII-ThDof, Supplementary Figure S3B and Figure 3B) into Arabidopsis leaves. Measurement of GUS activity and histochemical staining both demonstrated that activation of GUS in Arabidopsis cells when co-transformed with reporters containing complete motifs or the TheIF1A promoter-including motifs. However, when the reporter contained a mutated motif or TheIF1A promoter fragments without a motif, the Arabidopsis cells displayed less GUS staining and decreased GUS activity (Figures 3B,C). These findings clearly confirmed that ThDof may activate expression of TheIF1A by binding to the Dof motif present in its promoter.

The expression patterns of TheIF1A and ThDof in T. hispida under salt and drought stresses were analyzed by qRTPCR, and the results indicated that they share similar expression profiles. Under salt treatment, both genes exhibited peak transcription at $6 \mathrm{~h}$ in roots and stems, reaching peak levels in leaves at $24 \mathrm{~h}$ (Figure 1A). Under drought conditions, the lowest expression of both genes was observed 

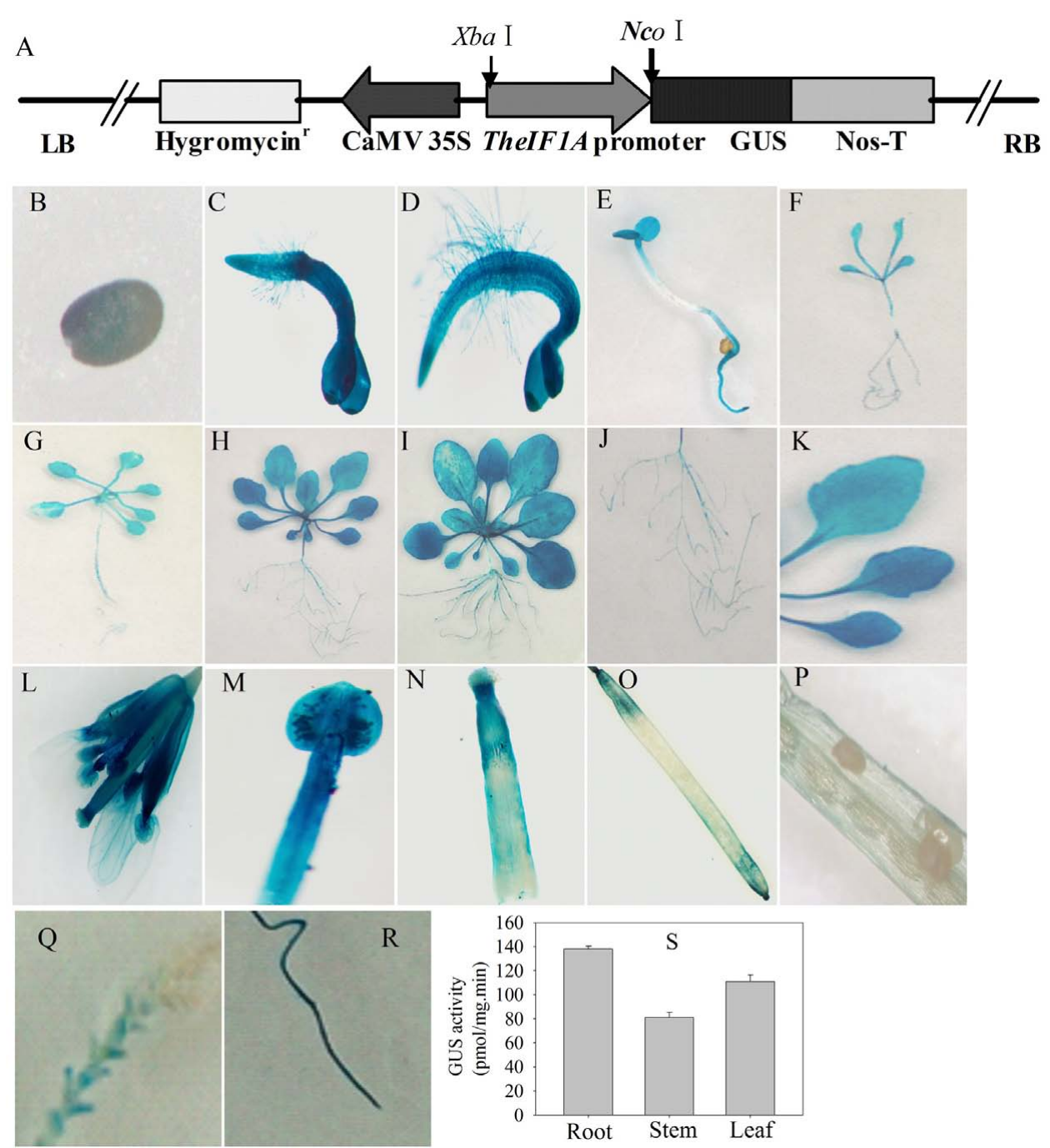

FIGURE 2 | Expression activity analysis of the TheIF1A promoter. Transgenic Arabidopsis plants carrying the ThelF1A promoter and transiently expressing T. hispida seedlings were used. (A) Schematic map of the ThelF1A promoter inserted into the pCAMBIA1301 binary vector, which was used for Arabidopsis transformation. (B-P) Expression of ThelF1A at different growth stages and in different organs or tissues. (B) Mature seed; (C) 3-days-old seedling; (D) 5-days-old seedling; (E) 7-days-old seedling; (F) 10-days-old seedling; (G) 14-days-old-seedling; (H) 3-week-old seedling; (I) 5-week-old seedling; (J,K) root and three rosette leaves from a 3-week-old plant; (L) whole flower cluster; (M) bracteole; (N) stigma; (O) intact fresh silique; (P) dissected silique; (Q) stem and leaves of T. hispida; (R), root of T. hispida; (S) GUS activity according to $(\mathbf{Q}, \mathbf{S})$. Data represent the means \pm SD of three independent experiments.

at $48 \mathrm{~h}$ in roots and stems. In leaves, both genes showed highest and lowest expression at 12 and $72 \mathrm{~h}$, respectively (Figure 1B).

\section{Overexpression of TheIF1A Improves Salt and Osmotic Stress Tolerance in Transgenic Tobacco}

As TheIF1A was induced by $\mathrm{NaCl}$ and PEG stresses, we investigated whether overexpression of TheIF1A in plants enhances salt and osmosis tolerance using 35S::TheIF1Atransgenic tobacco plants of the $\mathrm{T}_{3}$ generation. PCR analysis of the transgenic lines confirmed the successful transformation of TheIF1A into tobacco (Supplementary Figure S4A). The three lines of transgenic tobacco showing the highest expression were Lines 1, 3, and 9 (439.58-, 252.48-, and 508.46-fold compared with tubulin, respectively) (Supplementary Figure S4B), and these lines were selected for further analysis.

Germination rates were compared between the transgenic lines and wild type (WT) plants. Without treatment, there was no observable difference between WT and transgenic plants (Figure 4A). However, germination in the transgenic plants was significantly increased compared with WT under mannitol stress (Figures 4A,B) $(p<0.05)$. The water loss rates of the transgenic and WT plants (Figure 4C) were compared after leaves were exposed to air for $10 \mathrm{~min}$, and the rate of water loss in the transgenic lines was $83 \%$ of that in WT; after $70 \mathrm{~min}$, the water loss rate of the transgenic lines was also significantly decreased 


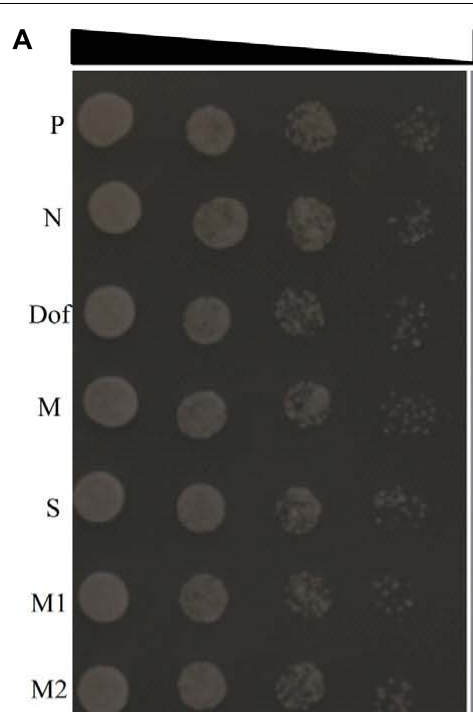

DDO

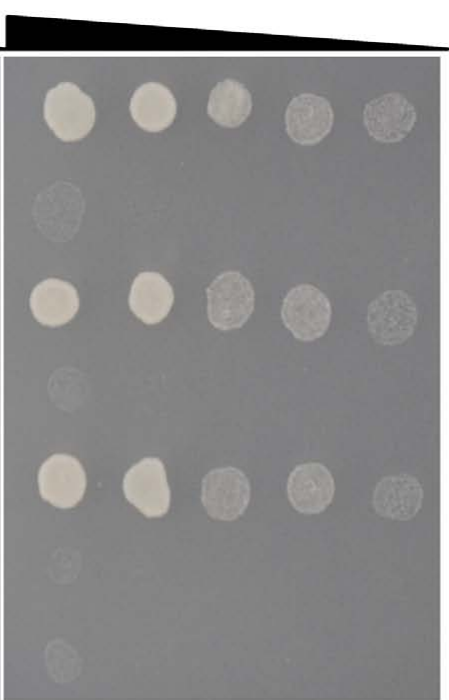

TDO/+50 mM 3-AT
B

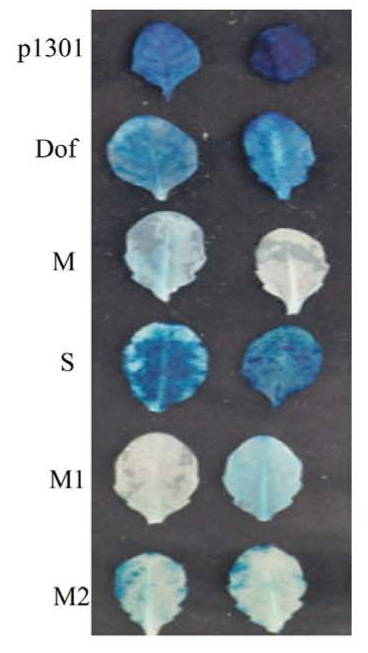

c

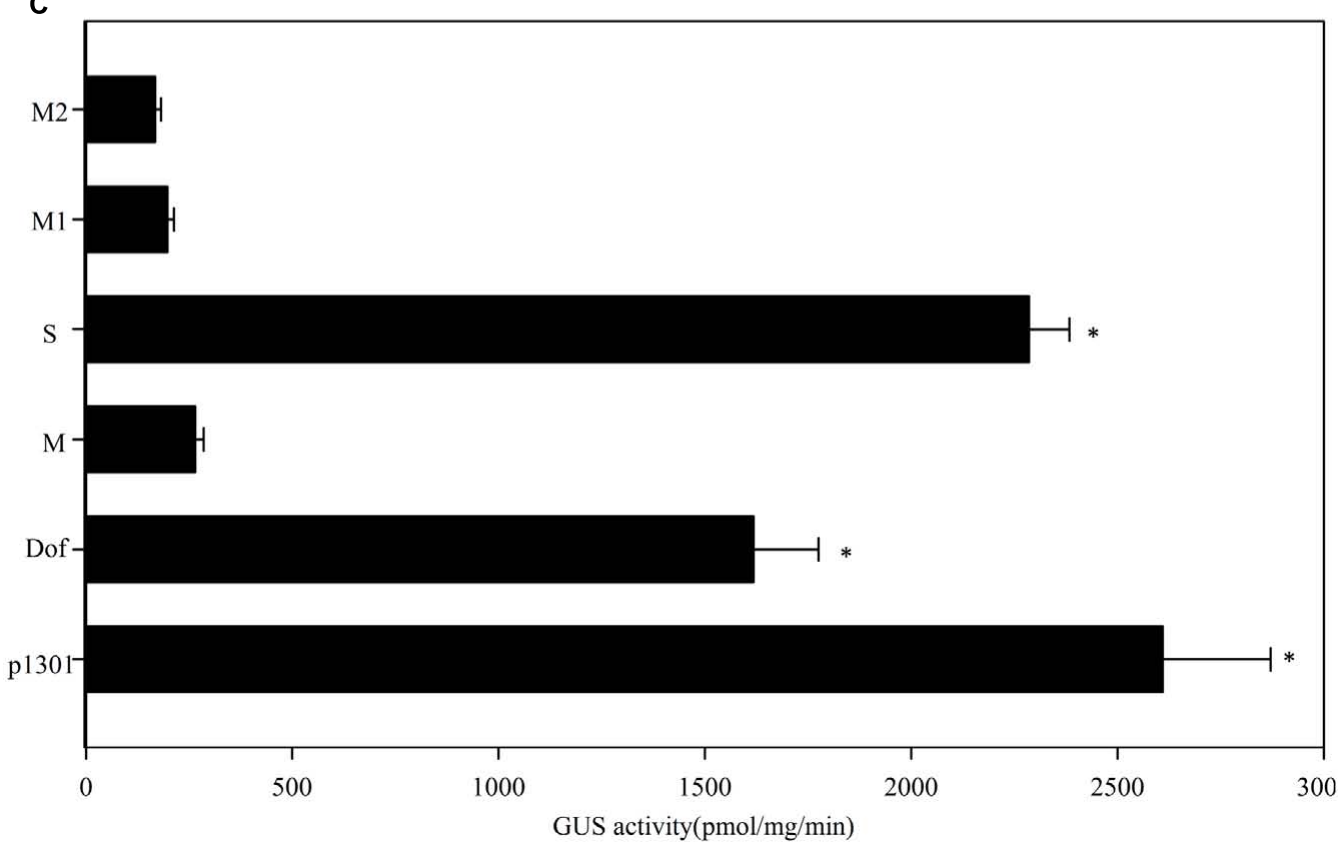

FIGURE 3 | Yeast one-hybrid analyses of upstream regulators of ThelF1A. (A) P, positive control, the pGADT7-Rec2 vector encoding murine p53 fused with GAL4 AD. N, negative control, the pHIS2 reporter vector containing the cis-acting DNA consensus sequence recognized by p53. Dof, Dof motif. M, indicates a mutated Dof motif. S, The ThelF1A promoter fragment containing the Dof motif. M1, The ThelF1A promoter fragment not containing the Dof motif. M2, The ThelF1A promoter fragment containing the mutated Dof motif. Transformants spotted onto SD/-Leu/-Trp (DDO) were used as positive controls for transformant growth. Positive transformants were further confirmed by spotting serial dilutions (1/1, 1/10, 1/100, 1/1000, 1/10000) onto SD/-His/-Leu/-Trp plates with 50 mM 3-AT (TDO/+50 mM 3-AT); the triangle indicates the dilutions from 1 to 10000 . (B) Results of transient reporter experiments for effector overexpression in Arabidopsis. p1301 and pCAMBIA1301 were used as positive controls. Dof, M, M1, and M2 were consistent with (A). (C) GUS activity according to (B). *indicates significant differences between the 'M' and other lines $(p<0.05)$.

(80\%) compared with WT plants. Although the chlorophyll content (tcc) of the transgenic plants was different from that in WT plants prior to salt or mannitol stress, tcc in all plants decreased strongly after 6 days of salt or mannitol stress. Nonetheless, the transgenic lines showed significantly higher tcc than WT plants (the difference was, on average, 1.20-1.26-fold)
(Figure 4D), which suggests that overexpression of TheIF1A prevents chlorophyll loss under salt or mannitol stress conditions.

To understand the regulation of physiological responses, physiological parameters involved in stresses were compared, including the ROS content, the activity of protective enzymes, 


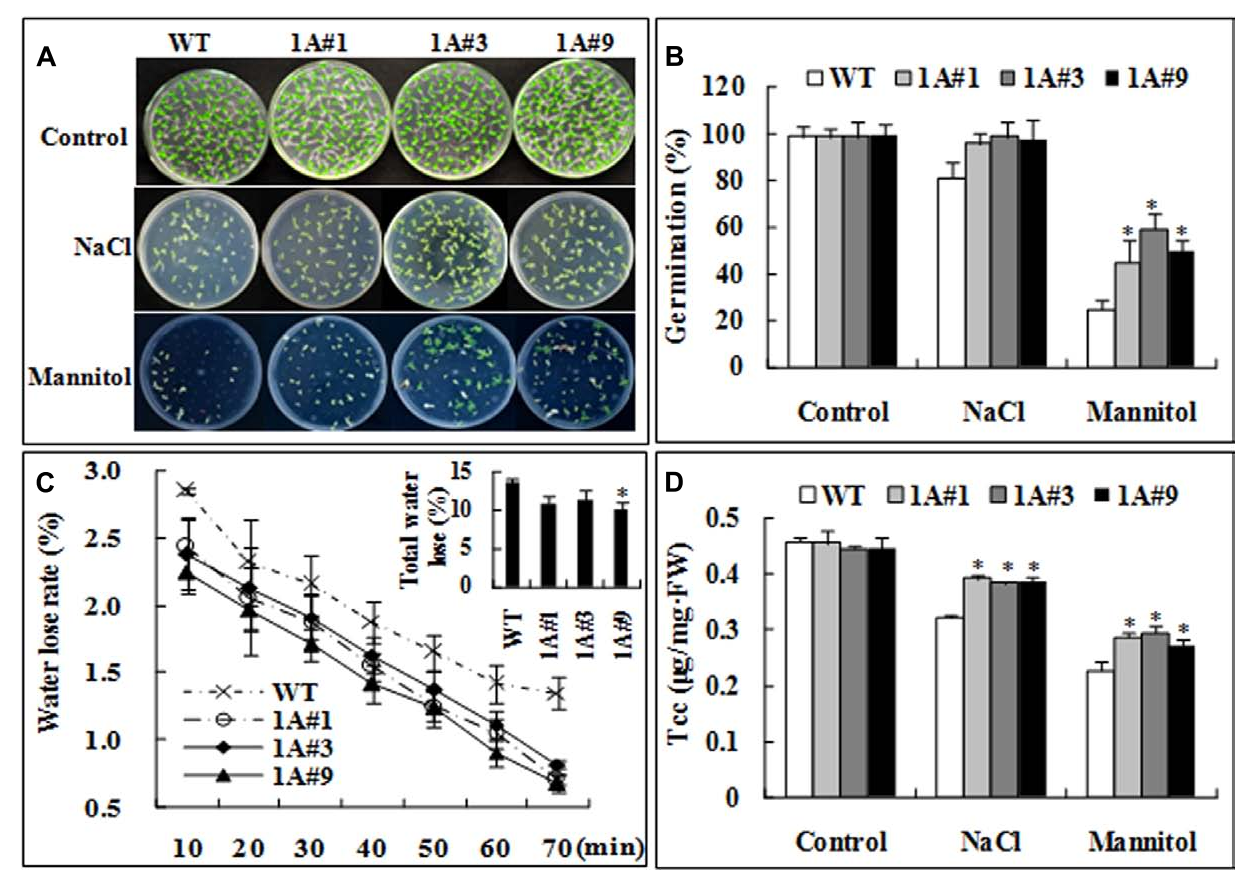

FIGURE 4 | Salt and osmotic stress tolerance analysis of WT and transgenic tobacco plants. WT, Wild type; 1A\#1, 1A\#3, and 1A\#9 are three transgenic ThelF1A lines. (A) Germination assay under $\mathrm{NaCl}$ and mannitol treatment for 12 days. (B)Germination percentage according to (A). (C) The weight of water loss from 28-days-old of WT and ThelF1A transgenic plants in $70 \mathrm{~min}$. (D) Total chlorophyll was compared after 6 days of NaCl or mannitol treatment of 28-days-old of WT and ThelF1A transgenic plants. The experiments were repeated at least three times, and 30 tobacco seedlings were used for each treatment. $*$ indicates significant differences between transgenic lines and WT $(p<0.05)$.

such as SOD, POD, GST and GPX, EL rates and the MDA content. Staining with diaminobenzidine (DAB) and nitroblue tetrazolium (NBT) demonstrated that $\mathrm{H}_{2} \mathrm{O}_{2}$ and $\mathrm{O}^{2-}$ levels, respectively, in leaves were similar in WT and transgenic lines under no treatment. However, WT plants exhibited increased $\mathrm{H}_{2} \mathrm{O}_{2}$ and $\mathrm{O}^{2-}$ accumulation compared with transgenic lines, particularly at $1 \mathrm{~h}$ after stress treatment, at which point the degree of staining in the transgenic lines was notably decreased compared with the WT plants (Figures 5A,B). ROS levels in intact guard cells and the main roots, as determined by stained with $\mathrm{H}_{2} \mathrm{DCF}$, were similar to the levels indicated by DAB and NBT staining. That is, with the exception of $0 \mathrm{~h}$, WT plants exhibited increased ROS accumulation in both guard cells and main roots compared with transgenic lines (Figures 5D,E). Additionally, the $\mathrm{H}_{2} \mathrm{O}_{2}$ content according to the degree of staining showed significantly less $\mathrm{H}_{2} \mathrm{O}_{2}$ in TheIF1A-transgenic plants than in WT plants (Figure 5F, $p<0.05$ ). These findings indicate that TheIF1A overexpression leads to a significant decrease in ROS accumulation in plant cells under salt and mannitol stress conditions.

Superoxide dismutase, POD, GST, and GPX are key ROS scavengers, and our results demonstrated similar changes in the activities of these enzymes. Under no treatment, no significant differences in the activities of these enzymes were observed between transgenic and WT plants. However, the activities of these enzymes in the transgenic plants were significantly $(p<0.05)$ increased compared with the WT plants under salt and mannitol stresses. For example, the SOD activity of the transgenic lines was, on average, approximately 1.4-fold that of WT under both stresses, and POD activity in the former was 1.41 -fold that of the latter under salt stress. GST activity of $1 \mathrm{A \# 3}$ was 2.16-fold that of WT exposed to $\mathrm{NaCl}$, and GPX activity of $1 \mathrm{A \# 3}$ was 1.63 - and 1.62 -fold of that of $\mathrm{WT}$ in response to $\mathrm{NaCl}$ and mannitol, respectively. Although the MDA content did not differ between transgenic lines and WT plants under no stress, the transgenic plants accumulated significantly less MDA compared with the WT plants after treatment. The average MDA content of WT was 1.40- and 1.23-fold of the transgenic lines under $\mathrm{NaCl}$ and mannitol stresses, respectively (Figure 6). These findings indicated that overexpression of TheIF1A increased enzyme activities, subsequently decreasing MDA accumulation.

In addition, the plasma membrane system was analyzed using Evans blue staining and EL rate determination. The tested lines did not exhibit apparent differences under control conditions. However, when treated with salt or mannitol, the transgenic plants exhibited weaker Evans blue staining and a lower EL rate compared with WT plants (the EL of WT was, on average, 1.33-fold higher than that of transgenic lines exposed to $\mathrm{NaCl}$ and 1.47-fold following mannitol), and these differences were significant (Figures 5C, 6).

Taken together, these findings suggested that TheIF1A overexpression improves salt and osmotic stress tolerance by increasing ROS scavenging and preventing cell damage to maintain growth. 

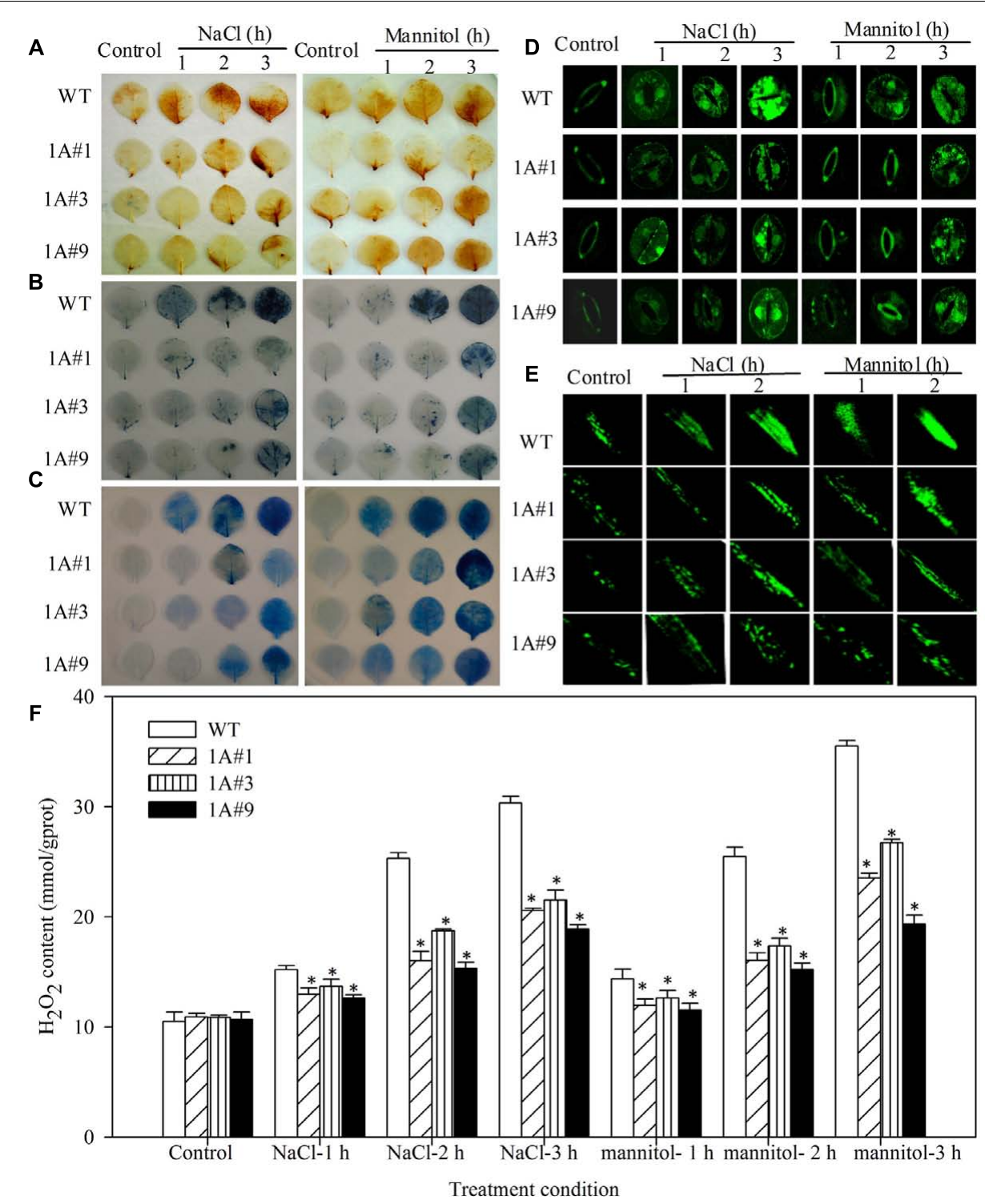

FIGURE 5 | Reactive oxygen species (ROS) levels and cell death in TheIF1A transgenic tobacco plants and WT under different stress conditions. All experiments were repeated at least three times, and approximately 15 leaves collected from multiple 28-days-old seedlings were inspected in each experiment. (A) DAB staining indicates accumulation of $\mathrm{H}_{2} \mathrm{O}_{2}$ in leaves of WT and transgenic plants subjected to 1, 2, or $3 \mathrm{~h}$ of salt or mannitol stress. (B) NBT staining of $\mathrm{O}^{2-}$ accumulation in leaves according to DAB staining. (C) Evans blue staining. (D) Representative microscopy images of ROS production in intact guard cells, as indicated by the fluorescent dye DCF. Epidermal peels were loaded with $\mathrm{H}_{2}$ DCF-DA for $10 \mathrm{~min}$ after incubation in fixing buffer for $2 \mathrm{~h}$. (E) ROS production in the root, as indicated by the fluorescent dye DCF. Main roots detached from control, $\mathrm{NaCl}$ and mannitol stress $(1 \mathrm{~h})$ plants were incubated in incubation buffer for $2 \mathrm{~h}$ at room temperature and subsequently stained with $5 \mu \mathrm{M} \mathrm{H}_{2}$ DCF-DA for 10 min. (F) The total $\mathrm{H}_{2} \mathrm{O}_{2}$ content according to the staining (A-F). *indicates significant differences between transgenic lines and WT $(p<0.05)$.

\section{Expression Analysis of Stress-related Genes in ThelF1A-transformed Tobacco Plants}

Tobacco plants overexpressing TheIF1A showed resistance to $\mathrm{NaCl}$ and mannitol stresses, and the activities of the corresponding protective enzymes were increased in transgenic lines compared with WT plants, suggesting that expression of key potential stress-related genes may be altered by exogenous expression of TheIF1A. Stress response-related genes were selected for analysis, and seven tobacco genes, including GST, MnSOD, NtMPK9, poxN1, CDPK15, and TOBLTP, exhibited increased levels of expression in TheIF1A-overexpressing lines compared with WT plants. In particular, levels of GST and MnSOD expression were 5.63- and 10.60-fold higher, respectively, than those in WT plants (Figure 7). These findings indicated that TheIF1A improves ROS scavenging via regulation of antioxidant genes. 


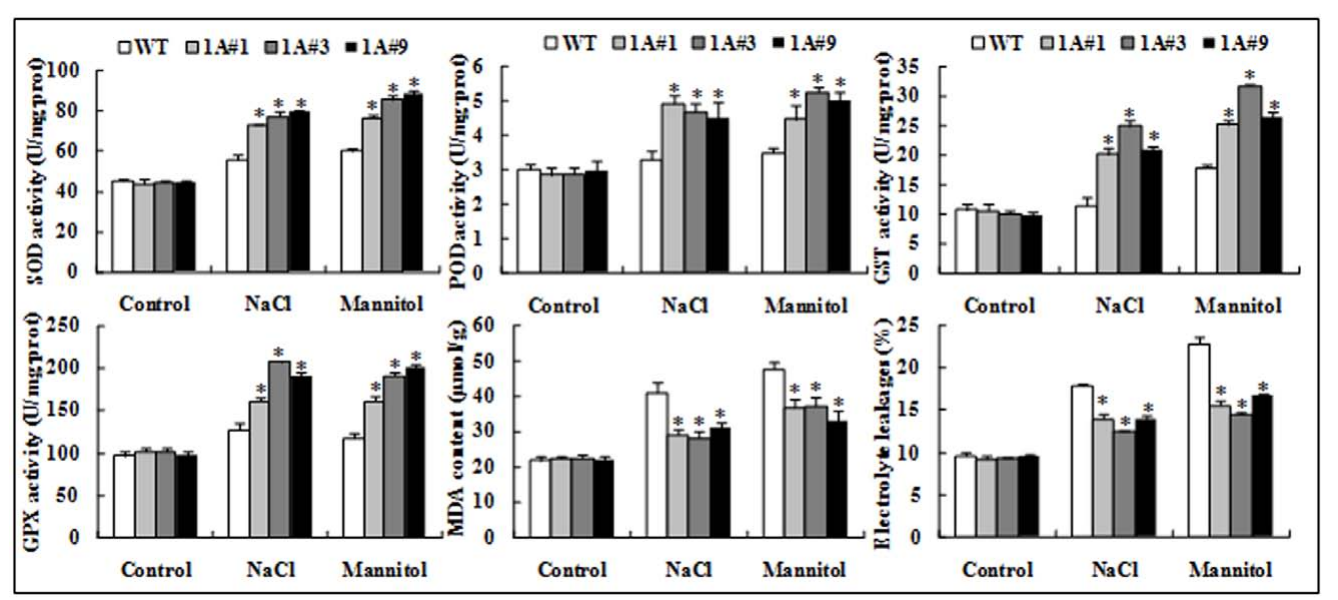

FIGURE 6 | Physiological index analyses of 28-days-old seedlings of ThelF1A transgenic plants and WT under $100 \mathrm{mM}$ NaCl or $200 \mathrm{mM}$ mannitol for 6 days; well-watered seedlings were used as the control. All experiments were repeated three times. Data represent the means \pm SD of three independent experiments. * Indicates significant differences between the transgenic lines and WT ( $p<0.05)$.

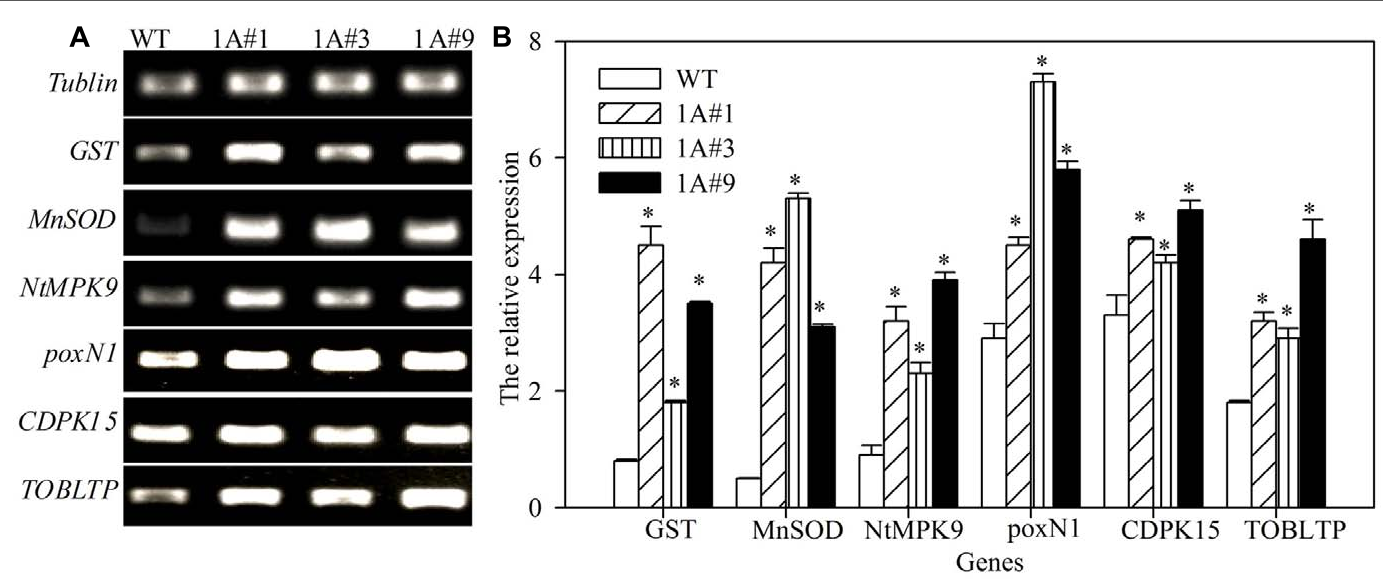

FIGURE 7 | Reverse-transcription-polymerase chain reaction (RT-PCR) analyses of stress-related genes in ThelF1A-transformed tobacco plants. (A) Gel electrophoresis of tobacco GST, MnSOD, poxN1, NtMPK9, CDPK15, and TOBLTP. Tubulin was used as the reference gene. (B) Relative expression level according to tubulin based on (A). All experiments were repeated three times. Data represent the means $\pm \mathrm{SD}$ of three independent experiments. ${ }^{*}$ Indicates significant differences between the transgenic lines and WT $(p<0.05)$.

\section{Transient Expression Analysis of ThelF1A in T. hispida}

To further confirm the role of TheIF1A in the T. hispida response to abiotic stress, 35S::TheIF1A and RNAi::TheIF1A were transiently transformed into $T$. hispida, and TheIF1A expression in two lines was determined by qRT-PCR. The results showed the highest TheIF1A expression in the 35S::TheIF1A plants and the lowest in the RNAi::TheIF1A plants. The level of TheIF1A expression in the overexpression line was 27.9-fold higher than that in the control plants and was 50.01-fold that in the RNAi::TheIF1A plants under no stress. These findings indicate the successful overexpression of TheIF1A in the 35S::TheIF1A plants and the RNAi-mediated silencing of TheIF1A in the RNAi::TheIF1A plants (Figure 8A). Interestingly, the expression patterns of ThSOD, ThPOD,
ThGSTZ1, and ThGPX were similar to that of TheIF1A. Expression of these genes was highest in 35S::TheIF1A lines and lowest in RNAi::TheIF1A lines (Figure 8A), which suggests that the abiotic stress response of TheIF1A may correlate with the level of ThSOD, ThPOD, ThGSTZ1, and ThGPX expression.

The related physiological indexes were determined. Under $\mathrm{NaCl}$ treatment, SOD, POD, GST, and GPX activities in the control plants were $49 \sim 77 \%$ of the activities in the $35 S::$ TheIF1A plants, and the activities of these enzymes in the RNAi::TheIF1A plants were $36 \sim 63 \%$ of the activities in the 35S::TheIF1A lines. After mannitol treatment, SOD, POD, GST, and GPX activities in the control plants were $56 \sim 77 \%$ of the activities in the 35S::TheIF1A plants; the activities of these enzymes in the RNAi::TheIF1A lines were $32 \sim 67 \%$ of the activities in the 

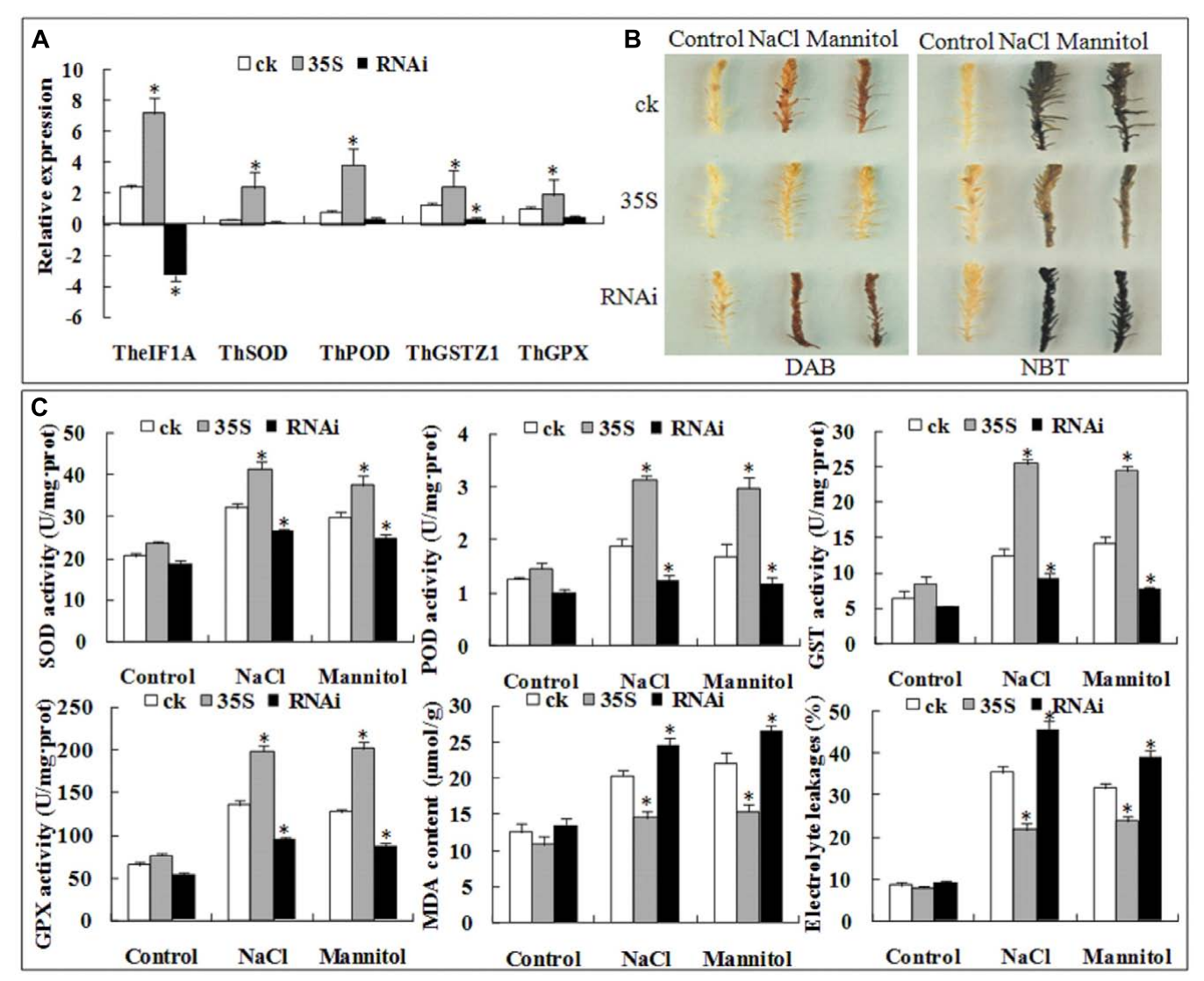

FIGURE 8| Transient expression analyses of TheIF1A in T. hispida under $100 \mathrm{mM}$ NaCl or $200 \mathrm{mM}$ mannitol treatments for $1 \mathrm{~h}$. ck, $35 \mathrm{~S}$, and RNAi indicate the empty pROKII, 35S::ThelF1A, and RNAi::ThelF1A-transformed lines, respectively. (A) Expression of ThelF1A, ThSOD, ThPOD, ThGSTZ1, and ThGPX in transient expression lines according to the reference gene. (B) DAB and NBT staining. (C) SOD, POD, GST, and GPX activities, the MDA content and EL of transiently expressing T. hispida seedlings. All experiments were repeated three times. Data represent the means $\pm \mathrm{SD}$ of three independent experiments.

* Indicates significant differences between pROKII and 35S::ThelF1A and between pROKII and RNAi::ThelF1A lines ( $p<0.05)$.

35S::TheIF1A seedlings. The MDA content in the 35S::TheIF1A plants was $71 \%$ of that in the control plants and $59 \%$ of that in the RNAi::TheIF1A plants under $\mathrm{NaCl}$ stress. After treatment with mannitol, the MDA content in the 35S::TheIF1A plants was 70\% of that in the control plants and $58 \%$ of that in the RNAi::TheIF1A lines. The EL rate in the $35 S::$ TheIF1A plants was $61 \%$ of that in the control plants and $48 \%$ of that in the RNAi::TheIF1A lines under $\mathrm{NaCl}$ stress. However, after exposure to mannitol, the EL rate in the $35 S::$ TheIF1A plants was $75 \%$ of that in the control plants and $61 \%$ of that in the RNAi::TheIF1A plants (Figure 8C), which indicates that TheIF1A effectively increased enzymatic activity and decreased cell damage.

Furthermore, DAB, NBT and Evans blue staining also demonstrated that 35S::TheIF1A T. hispida seedlings accumulated less ROS and suffered less cell damage and that RNAi::TheIF1A plants accumulated more ROS and suffered more cell damage compared with control plants after treatment with $100 \mathrm{mM} \mathrm{NaCl}$ or $200 \mathrm{mM}$ mannitol (Figure 8B). Taken together, these findings indicate that TheIF1A improved plant tolerance via regulation of other stress-related genes, the management of ROS homeostasis, and the prevention of cell damage and death.

\section{DISCUSSION}

Some reports have indicated the involvement of $\operatorname{eIF1A}$ in the response to abiotic stress. For example, sugar beet BveIF1A not only partially complemented a yeast eIF1A-deficient strain but also increased the $\mathrm{NaCl}$ tolerance of BveIF1A transgenic Arabidopsis plants (Rausell et al., 2003). The assembly, kinetics and composition of stress granules in yeast may also be related to (eIF)3, eIF4A/B, eIF5B, and eIF1A proteins (Buchan et al., 2011). Expression of LceIF1 from Leymus chinensis (Trin.) was decreased under sodium-saline stresses, whereas, overexpression was induced under sodic-alkaline stresses; however, when expressed in most organisms under no treatment, eIF1-transgenic lines exhibited relatively high eIF1 expression, which resulted in potentially increased stress resistance (Sun and Hong, 2013). These findings indicate that $\operatorname{eIF1}(A)$ may also play a positive role in response to abiotic stress tolerance. Thus, the eIF1A gene from the halophyte T. hispida warrants investigation under exposure to abiotic stress. Here, we characterized TheIF1A in plants exposed to salt and drought, and our results provide a new and further perspective on the function of $\operatorname{eIF} 1(A)$ in stress responses. 


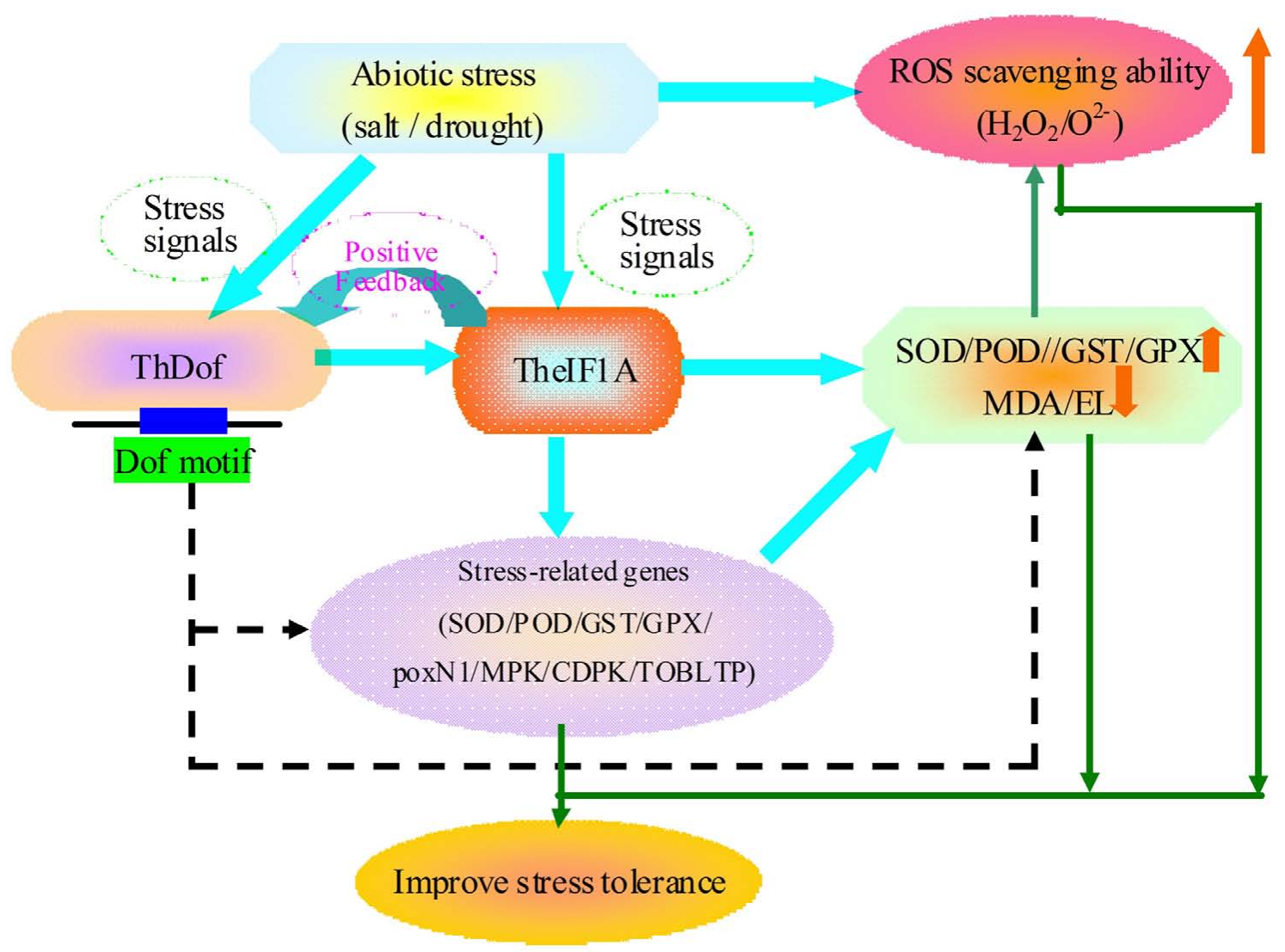

FIGURE 9 | Mapping of the potential tolerance regulation mechanism of TheIF1A. The solid line with arrows is based on our results, whereas, the dotted line with arrows is speculation based on the current and previous studies.

In the present study, TheIF1A expression in T. hispida was induced in roots, stems, and leaves after treatment of plants with $\mathrm{NaCl}$ and PEG. In a previous study, Diédhiou et al. (2008) demonstrated that eIF1 transcription was only increased in salt-tolerant plants after prolonged salt treatment, whereas, the expression levels in salt-sensitive plants were not induced. The eIF1 gene from the salt-tolerant plant Porteresia coarctata was only induced after treatment with $\mathrm{NaCl}$ for 3 and 5 days (Latha et al., 2004). In addition, BveIF1A was not induced under $\mathrm{NaCl}$ stress for $24 \mathrm{~h}$, whereas, AteIF1A expression was slightly decreased under the same stress (Rausell et al., 2003). Our results indicate that TheIF1A is induced by $\mathrm{NaCl}$ and PEG stress and that TheIF1A may play a potential role in salt and PEG tolerance.

The results of TheIF1A promoter GUS staining showed that TheIF1A was expressed throughout all plant parts at every developmental stage, with the exception of fresh siliques and seeds. Transient transformation of the TheIF1A promoter into $T$. hispida seedlings also resulted in different tissue expression patterns. Conversely, Wang et al. (2012) indicated that TaeIF5A1 exhibited similar expression patterns in different tissues and growth stages, which suggests that TaeIF5A1 may function in stress tolerance via gene regulation in all tissues. TaeIF3g was induced in leaves under drought stress, and TaeIF3g overexpression improved plant drought tolerance (Singh et al.,
2007). Several other $\mathrm{eIFs}$ are also reportedly regulated in response to different abiotic stresses (Costa-Neto et al., 2006; Wang et al., 2012). However, there are few reports on the role of $e I F 1 A$ in abiotic stress responses based on physiological regulation, such as tcc, protective enzymes, and ROS levels.

Accordingly, TheIF1A was overexpressed in tobacco to further understand the involvement of this gene in stress resistance. Germination, hydration, and chlorophyll production are important for normal plant growth, and these processes are disrupted during stress. Specifically, the water content, chlorophyll level and net photosynthetic rate are vital for plant development. The tcc level of TaeIF5A- or ThWRKY4overexpressing Arabidopsis was increased compared with that of WT plants, suggesting that TaeIF5A and ThWRKY4 regulate salt tolerance genes (Wang et al., 2012; Zheng et al., 2013). In the present study, TheIF1A was associated with these indicators, and tobacco plants overexpressing TheIF1A exhibited increased germination rates, decreased water loss rates and more stable tcc compared with WT and control plants under salt or mannitol stresses; these results suggest that TheIF1A may participate in physiological regulation in plants. Furthermore, the potential ROS metabolism system was examined to verify the regulation mechanism of TheIF1A, and the results indicated that TheIF1A overexpression positively enhanced 
the activities of plant protective enzymes and reduced ROS accumulation under $\mathrm{NaCl}$ or mannitol stresses. Taken together, the current findings suggest that TheIF1A expression improves the activity of antioxidant enzymes, thereby enhancing ROS scavenging.

Previous studies have indicated that compared with WT plants, $M n S O D$ overexpression effectively improved SOD activity and salt stress tolerance (Tanaka et al., 1999). Plant POD genes are involved in several different physiological functions, including growth regulation and wound healing; many poxN genes are wound inducible and display low induction under no treatment, whereas, expression of these genes is rapidly induced after exposure to abiotic stress (Ito et al., 2000). GSTs are a superfamily of multifunctional, dimeric enzymes that participate in detoxification of endo- and xenobiotics, and some GST genes have been implicated in responses to dehydration (Kiyosue et al., 1993; Bianchi et al., 2002), hydrogen peroxide $\left(\mathrm{H}_{2} \mathrm{O}_{2}\right)$ and salicylic acid (SA) signaling (Chen et al., 1996), herbicide application (Edwards et al., 2000), wounding (Vollenweider et al., 2000), auxin production (Chen and Singh, 2002), and salt stress (Moons, 2003). In a previous study, we demonstrated that ThGSTZ1 effectively improves plant resistance to salt and mannitol stresses (Yang et al., 2014). MPK is a member of the MKK2 pathway, which mediates cold and salt stress signaling in Arabidopsis (Teige et al., 2004). Popescu et al. (2009) demonstrated that MPK phosphorylation enriched TFs involved in regulating development, defense, and stress in plants. As osmotic stress induces calcium signaling, CDPK (calcium-dependent protein kinase) genes are prime candidates as the link between calcium signaling and downstream responses (Zhu, 2002). CDPK activates a stress-inducible promoter, which bypasses the stress signals that have various functions mediated through different CDPKs, suggesting that these factors act as potential positive regulators that control stress signal transduction in plants (Sheen, 1996). TOBLTP encodes a lipid transfer protein, the expression of which is triggered by ABA and drought (Torres-Schumann et al., 1992; Treviño and O'Connell, 1998). Considering that the activity of several protective enzymes is enhanced, it is important to determine the expression profiles of these genes in TheIF1Aoverexpressing plants. The induced expression of GST, MnSOD, NtMPK9, poxN1, CDPK15, and TOBLTP in TheIF1A-transgenic tobacco plants suggests that TheIF1A may be involved in the regulation of these genes to enhance abiotic stress tolerance. Furthermore, transient expression of TheIF1A in T. hispida seedlings increased expression of ThSOD, ThPOD, ThGSTZ1, and ThGPX in 35S::TheIF1A plants, whereas it reduced expression of these genes in RNAi::TheIF1A lines. Moreover, all plants exhibited increased expression levels under $\mathrm{NaCl}$ or mannitol treatment. These findings suggest that all of these genes are responsive to stress, providing candidate regulators in stress regulation pathways. The results of these analyses suggested that TheIF1A regulates other genes, such as SOD and POD, to enhance related activities, increase ROS scavenging and decrease ROS accumulation.

Many motifs, such as DOFCOREZM, MYBCORE, and WRKY71OS, were identified in the promoter of TheIF1A, a finding that suggests the role of TheIF1A in the response to abiotic stress involves different TFs. Dof TFs are important positive regulators in plant responses to adverse stimuli. Tomato $D O F$ genes exhibit distinct diurnal expression patterns and are differentially induced in response to osmotic, salt, heat, and lowtemperature stresses. SlCDF1- or SlCDF3-transgenic Arabidopsis plants exhibit increased drought and salt tolerance (Corrales et al., 2014). The expression profiles of nine BraDof family genes under cold, heat, salt, and drought treatments in 'Lubaisanhao' and 'Qingdao 87-114' were examined by qRT-PCR, and most were up-regulated (Ma et al., 2015). Thus, we predict that TheIF1A may respond to salt and drought stresses in a manner similar to that of Dof genes. Zheng et al. (2013) reported that ThDof is an upstream regulator of ThWRKY4 in the regulation of ThWRKY4 expression and function. Dof may also interact with and stimulate the DNA binding of stress-responsive bZIP proteins (Kang and Singh, 2000). Many Dof domain proteins enhance growth and tolerance to dehydration and salt stress (Foyer and Noctor, 2005; Shukla et al., 2006). Based on these previous studies and the current results obtained from yeast onehybrid assays and expression analyses, we speculate that ThDof is an upstream regulator of TheIF1A that may regulate induction of the latter by binding to the Dof motif in its promoter and that these two proteins may function in the same stress regulation pathway.

\section{CONCLUSION}

We demonstrated that TheIF1A acts as a stress response regulator to improve plant salt and osmosis tolerance via the maintenance of tcc and by increasing the activity of protective enzymes, decreasing ROS generation and regulating other related genes to improve the ROS scavenging ability and reduce cell damage (Figure 9). Furthermore, ThDof specially binds to the Dof motif in the TheIF1A promoter and may regulate or interact with TheIF1A to participate in salt and osmotic stress responses in plants.

\section{MATERIALS AND METHODS}

\section{Plant Materials and Treatments}

Tamarix hispida and Arabidopsis thaliana (Col, WT) seedlings were cultivated in pots containing a mixture of turf peat and sand $(2: 1 \mathrm{v} / \mathrm{v})$ in a greenhouse for 2 months under $14 \mathrm{~h}$ light/10 h dark, 70-75\% relative humidity, an average temperature of $24^{\circ} \mathrm{C}$ and well-watered conditions. Two-monthold T. hispida seedlings were exposed to the following treatments: $400 \mathrm{mM} \mathrm{NaCl}$ or $20 \%(\mathrm{w} / \mathrm{v}) \mathrm{PEG}_{6000}$ for $6,12,24,48$, or $72 \mathrm{~h}$. The roots, stems, and leaves from at least 20 seedlings were then independently harvested at the indicated time points after each treatment and pooled for qRT-PCR analyses. Seedlings irrigated with water alone were used as the control; every treatment was carried out three times as biological replicates, with every replicate containing at least 20 seedlings. 


\section{Cloning of the ThelF1A Gene and its Promoter}

The TheIF1A gene (KF801668) was cloned from the T. hispida leaf cDNA library (Gao et al., 2008) The phylogenetic tree of eIF1A proteins from different plant species was constructed using the Neighbor-Joining method (Saitou and Nei, 1987). The TheIF1A promoter was PCR amplified from T. hispida genomic DNA using a genome walking kit. cis-elements in the TheIF1A promoter were identified using the PLACE database ${ }^{1}$ (Higo et al., 1999). $T_{3}$ seedlings of TheIF1A promoter transgenic line were used for temporal and spatial expression analyses of TheIF1A via GUS staining (Clough and Bent, 1998). Moreover, pCAMBIA1301-TheIF1A was transiently expressed in T. hispida seedlings to verify the TheIF1A expression results obtained in A. thaliana.

\section{RNA Isolation and qRT-PCR}

Total RNA was isolated from seedlings exposed to different treatments using the CTAB method (Wang et al., 2002); $0.5 \mu \mathrm{g}$ of RNA was used for reverse transcription with PrimeScript ${ }^{\mathrm{TM}}$ RT reagent Kit (Takara). The resulting cDNA product was diluted 10-fold and used for RT-PCR analyses. qRT-PCR was applied using an MJ Opticon ${ }^{\mathrm{TM} 2}$ machine (Bio-Rad, Hercules, CA, USA); the reaction system and procedures were performed according to Gao et al. (2011). $\beta$-Actin (FJ618517), $\alpha$-tubulin (FJ618518), and $\beta$-tubulin (FJ618519) were used as reference genes. The primer sequences are listed in Supplementary Table S1. Relative expression levels were calculated using the deltadelta Ct method (Livak and Schmittgen, 2001). The RT-PCR data for the transgenic tobacco lines were normalized to tubulin (AJ421412) (Ban et al., 2011). Three independent experiments were performed, and at least 20 seedlings were used in each treatment.

\section{Identification of the Upstream Regulator of ThelF1A}

Twelve Dof motifs (core base sequence "AAAG") were identified in the TheIF1A promoter (Supplementary Figure S2). A yeast one-hybrid assay (Clontech, Palo Alto, CA, USA) was used to investigate specific genes recognizing these motifs and activating TheIF1A expression. The three tandem copies of the promoter fragment containing the Dof motif core ("AAAAGT") sequence were cloned into the pHIS2 vector (Supplementary Figures $\mathrm{S} 3 \mathrm{~A}, \mathrm{~B}$ ). TFs from different families were identified, PCR amplified and subsequently cloned into pGADT7-Rec2 (Clontech) to produce a cDNA library used in the one-hybrid assay (Zheng et al., 2013).

To determine interactions between the Dof motif and related positive clones, a mutated Dof core motif "AAAG" with "CCCT" (Dof-M) was constructed. Furthermore, fragments of the TheIF1A promoter containing the Dof motif (Dof-S), excluding the Dof motif (Dof-S-M1) or containing a mutated Dof motif (Dof-S-M2) were cloned into pHIS2 (Supplementary Figure S3B). Cells transformed with the empty p53HIS2 plasmid

\footnotetext{
${ }^{1}$ http://bioinformatics.psb.ugent.be/webtools/plantcare/html/
}

(pHIS2 contains three copies of the p53 DNA element) were used as a negative control in the yeast one-hybrid assay. All primers are presented in Supplementary Table S2.

To confirm these interactions, all constructs were fused with a CaMV35S-46 minimal promoter and cloned into pCAMBIA1301 for expression of the GUS gene. The fulllength cDNA of ThDof was cloned into pROKII under the control of the $35 \mathrm{~S}$ promoter (referred to as pROKII-ThDof) to create effecter vectors (Supplementary Figure S3C), which were transformed into Arabidopsis using the floral dip method (Clough and Bent, 1998). A $\mathrm{T}_{3}$ generation line of ThDof-transgenic Arabidopsis was then used for transient expression of all Dof reporters using Agrobacterium-mediated transformation. All cotransformed Arabidopsis leaves were stained to measure GUS activity (Jefferson et al., 1987; Jefferson, 1989). Furthermore, the expression patterns of TheIF1A and ThDof under salt and drought conditions in T. hispida were analyzed by qRT-PCR.

\section{Generation of Transgenic Tobacco}

The full-length cDNA of TheIF1A was amplified and cloned into pROKII (referred to as 35S::TheIF1A). The primer sequences are presented in Supplementary Table S2. 35S::TheIF1A was transferred into tobacco (Nicotiana tabacum) using Agrobacterium-mediated transformation. Sixty-one lines were generated in the $\mathrm{T}_{0}$ generation, which were further selected until the $\mathrm{T}_{3}$ generation; 12 different lines were detected by PCR, and the expression levels were analyzed by qRT-PCR. Three transgenic lines (Lines 1, 3, and 9, referred to as $1 \mathrm{~A} \# 1,1 \mathrm{~A} \# 3$, and $1 \mathrm{~A} \#$, respectively) with the highest expression levels were selected for further analysis.

\section{Stress Tolerance Analysis}

For the seed germination assay, seeds from WT and transgenic lines were sown onto 1/2 MS agar medium containing either $100 \mathrm{mM} \mathrm{NaCl}$ or $200 \mathrm{mM}$ mannitol. Germination was recorded after 12 days using germination rate $=$ germinated plantlets number/total seeds sown*100\%. For stress tolerance assays, 28-days-old WT and transgenic plants were treated with $100 \mathrm{mM} \mathrm{NaCl}$ or $200 \mathrm{mM}$ mannitol for 6 days. Leaves were used to determine tcc, SOD activity, POD activity, GPX activity, GST activity and the MDA content using a corresponding commercially available kit from Nanjing Jiancheng Bioengineering Institute (Nanjing, China). SOD activity was assessed based on the auto-oxidation of hydroxylamine. POD activity was tested using a peroxidase assay kit, with absorbance at 340 and $420 \mathrm{~nm}$. GST activity was determined using a glutathione $S$-transferase (GSH-ST) assay kit (Colorimetric method). GPX (GSH-Px) activity was determined by the velocity method using a GSH-Px kit; the reaction was initiated by the addition of $\mathrm{H}_{2} \mathrm{O}_{2}$, and the change in absorbance during the conversion of GSH to GSSG was recorded spectrophotometrically at $412 \mathrm{~nm}$. The MDA content was determined based on thiobarbituric acid (TBA) reactivity; the developed red color of the resulting reaction was measured at $532 \mathrm{~nm}$ using a spectrophotometer. The $\mathrm{H}_{2} \mathrm{O}_{2}$ content was assessed on the basis of a hydrogen peroxide assay kit (Minotti et al., 1994; Kwak et al., 2005; Ban et al., 2011; Liu et al., 2013). 
The EL rate was evaluated per the report of Yang et al. (2014). For water-holding ability, the aerial parts of seedlings were placed onto clean filter paper and dried for $70 \mathrm{~min}$. The fresh weights of the samples were measured every $10 \mathrm{~min}$ to determine the rate of water loss relative to each time interval.

In addition, leaves from seedlings treated with $100 \mathrm{mM}$ $\mathrm{NaCl}$ or $200 \mathrm{mM}$ mannitol for 0 (control), 1, 2, and $3 \mathrm{~h}$ were detached and immediately used for histochemical staining analysis. $\mathrm{O}^{2-}$ accumulation, $\mathrm{H}_{2} \mathrm{O}_{2}$ accumulation, and cell death in the leaves were assayed through NBT, DAB, and Evans blue staining, respectively. ROS production in intact guard cells and roots was assessed by staining with $5 \mu \mathrm{M} \mathrm{H} \mathrm{H}_{2}$ DCF-DA (Fluka) (Fryer et al., 2002; Zhang et al., 2011). ROS accumulation was visualized using an LSM410 confocal laser scanning microscope (CLSM) (Zeiss, Jena, Germany) with excitation at $488 \mathrm{~nm}$ and an emission at $525 \mathrm{~nm}$, and images were acquired using ZEN 2009 light edition imaging software (Zhang et al., 2011). The corresponding $\mathrm{H}_{2} \mathrm{O}_{2}$ content under the same treatments as staining assays was also examined. Furthermore, stress-related genes (TOBLTP, GST, MnSOD, NtMPK9, poxN1, and CDPK15) in TheIF1A-overexpressing tobacco plants were investigated by RT-PCR using the primers presented in Supplementary Table S3. All experiments were performed three times.

\section{Transient Expression of ThelF1A in T. hispida}

To better analyze the salt and mannitol response of TheIF1A, 295-bp of the TheIF1A sequence was cloned into a reconstructive ProKII vector to construct the RNAi-suppression vector RNAi::TheIF1A (Supplementary Figure S5) (Zhang et al., 2012; Li et al., 2014). The forward and reverse primers are presented in Supplementary Table S2. A single colony of EHA105 harboring 35S::TheIF1A, RNAi::TheIF1A and the empty ProKII vector (used as a control, labeled ck) was cultivated at $28^{\circ} \mathrm{C}$ to $\mathrm{OD}_{600}=0.6$, after which the cells were harvested by centrifugation and adjusted to $\mathrm{OD}_{600}=0.2$ using $1 / 2 \mathrm{MS}$ solution $(\mathrm{pH}=5.8)$ containing $3 \%(\mathrm{w} / \mathrm{v})$ sucrose, as the transformation solution supplied with $150 \mu \mathrm{M}$ acetosyringone. Two-month-old T. hispida seedlings were soaked in the transformation solution for $48 \mathrm{~h}$ at $25^{\circ} \mathrm{C}$ and 30-40 rpm, and the culture solution was changed every $4 \mathrm{~h}$ (Zheng et al., 2012). The transformed seedlings were treated

\section{REFERENCES}

Ban, Q., Liu, G., and Wang, Y. (2011). A DREB gene from Limonium bicolor mediates molecular and physiological responses to copper stress in transgenic tobacco. J. Plant Physiol. 168, 449-458. doi: 10.1016/j.jplph.2010.08.013

Battiste, J. L., Pestova, T. V., Hellen, C. U., and Wagner, G. (2000). The eIF1A solution structure reveals a large RNA-binding surface important for scanning function. Mol. Cell 5, 109-119. doi: 10.1016/S1097-2765(00) 80407-4

Bianchi, M. W., Roux, C., and Vartanian, N. (2002). Drought regulation of GST8, encoding the Arabidopsis homologue of ParC/Nt107 glutathione transferase/peroxidase. Physiol. Plant. 116, 96-105. doi: 10.1034/j.1399-3054. 2002.1160112.x

Buchan, J. R., Yoon, J. H., and Parker, R. (2011). Stress-specific composition, assembly and kinetics of stress granules in Saccharomyces cerevisiae. J. Cell Sci. 124, 228-239. doi: 10.1242/jcs.078444 with $100 \mathrm{mM} \mathrm{NaCl}$ or $200 \mathrm{mM}$ mannitol for $1 \mathrm{~h}$. DAB, NBT and Evans blue staining was subsequently used to visualize the $\mathrm{O}^{2-}$ and $\mathrm{H}_{2} \mathrm{O}_{2}$ contents and cell damage in the leaves; POD, SOD, GST, GPX activities and the MDA content were also examined. In addition, the expression levels of stress-related genes and ThSOD, ThPOD, ThGSTZ1, and ThGPX in seedlings of these three transgenic lines were examined by qRT-PCR. The primers are presented in Supplementary Table S1. All experiments were performed three times. Every independent replicate included at least 12 seedlings per line.

\section{Statistical Analysis}

All data were analyzed using Statistical Package for Social Science (SPSS) (SPSS, Chicago, IL, USA). Differences among the tested lines were calculated using Tukey's multiple comparison tests. The significance level was set as $p<0.05$, and the standard deviation (SD) was calculated to analyze sample variability.

\section{AUTHOR CONTRIBUTIONS}

GY wrote the manuscript and performed some of the assays (data shown in Figures 2, 3, 5, 8, 9). LY performed most of the assays (obtained the transgenic tobacco plants and cloned the promoter; data shown in Figures 1, 4, 6, 7). YW and CW performed the data analysis and also revised the manuscript. CG provided funds for the current study, designed the study and revised the manuscript.

\section{ACKNOWLEDGMENTS}

This work was supported by the National Natural Science Foundation of China (No. 31370676), the Fundamental Research Funds for the Central Universities (No. 2572014DA03), and New Century Excellent Talents in University (No. NCET-13-0709).

\section{SUPPLEMENTARY MATERIAL}

The Supplementary Material for this article can be found online at: http://journal.frontiersin.org/article/10.3389/fpls.2017.00513/ full\#supplementary-material

Chen, W., Chao, G., and Singh, K. B. (1996). The promoter of a H2O2-inducible, Arabidopsis glutathione S-transferase gene contains closely linked OBF-and OBP1-binding sites. Plant J. 10, 955-966. doi: 10.1046/j.1365-313X.1996. 10060955.x

Chen, W., and Singh, K. B. (2002). The auxin, hydrogen peroxide and salicylic acid induced expression of the Arabidopsis GST6 promoter is mediated in part by an ocs element. Plant J. 19, 667-677. doi: 10.1046/j.1365-313x.1999.00560.x

Clough, S. J., and Bent, A. F. (1998). Floral dip: a simplified method for Agrobacterium-mediated transformation of Arabidopsis thaliana. Plant J. 16, 735-743. doi: 10.1046/j.1365-313x.1998.00343.x

Corrales, A. R., Nebauer, S. G., Carrillo, L., Fernandez-Nohales, P., Marques, J., Renau-Morata, B., et al. (2014). Characterization of tomato cycling Dof factors reveals conserved and new functions in the control of flowering time and abiotic stress responses. J. Exp. Bot. 65, 995-1012. doi: 10.1093/jxb/ert451

Costa-Neto, C. M., Parreiras-e-Silva, L. T., Ruller, R., Oliveira, E. B., Miranda, A., Oliveira, L., et al. (2006). Molecular modeling of the human 
eukaryotic translation initiation factor 5A (eIF5A) based on spectroscopic and computational analyses. Biochem. Biophys. Res. Commun. 347, 634-640. doi: 10.1016/j.bbrc.2006.06.119

Diédhiou, C., Popova, O., Dietz, K. J., and Golldack, D. (2008). The SUIhomologous translation initiation factor eIF-1 is involved in regulation of ion homeostasis in rice. Plant Biol. 10, 298-309. doi: 10.1111/j.1438-8677.2008. 00037.x

Dong, Z., and Zhang, J. (2006). Initiation factor eIF3 and regulation of mRNA translation, cell growth, and cancer. Crit. Rev. Oncol. Hematol. 59, 169-180. doi: 10.1016/j.critrevonc.2006.03.005

Edwards, R., Dixon, D. P., and Walbot, V. (2000). Plant glutathione-S-transferases: enzymes with multiple functions in sickness and in health. Trends Plant Sci. 5, 193-198. doi: 10.1016/S1360-1385(00)01601-0

Foyer, C. H., and Noctor, G. (2005). Oxidant and antioxidant signalling in plants: a re-evaluation of the concept of oxidative stress in a physiological context. Plant Cell Environ. 28, 1056-1071. doi: 10.1111/j.1365-3040.2005.01327.x

Fryer, M. J., Oxborough, K., Mullineaux, P. M., and Baker, N. R. (2002). Imaging of photo-oxidative stress responses in leaves. J. Exp. Bot. 53, 1249-1254.

Gao, C., Wang, Y., Jiang, B., Liu, G., Yu, L., Wei, Z., et al. (2011). A novel vacuolar membrane $\mathrm{H}+$-ATPase $\mathrm{c}$ subunit gene (ThVHAc1) from Tamarix hispida confers tolerance to several abiotic stresses in Saccharomyces cerevisiae. Mol. Biol. Rep. 38, 957-963. doi: 10.1007/s11033-010-0189-9

Gao, C., Wang, Y., Liu, G., Yang, C., Jiang, J., and Li, H. (2008). Expression profiling of salinity-alkali stress responses by large-scale expressed sequence tag analysis in Tamarix hispida. Plant Mol. Biol. 66, 245-258. doi: 10.1007/s11103-0079266-4

Groušl, T., Ivanov, P., Frydlová, I., Vašicová, P., Janda, F., Vojtová, J., et al. (2009). Robust heat shock induces eIF2 $\alpha$-phosphorylation-independent assembly of stress granules containing eIF3 and 40S ribosomal subunits in budding yeast, Saccharomyces cerevisiae. J. Cell Sci. 122, 2078-2088. doi: 10.1242/jcs.045104

Higo, K., Ugawa, Y., Iwamoto, M., and Korenaga, T. (1999). Plant cis-acting regulatory DNA elements (PLACE) database: 1999. Nucleic Acids Res. 27, 297-300. doi: 10.1093/nar/27.1.297

Howarth, C. (1991). Molecular responses of plants to an increased incidence of heat shock. Plant Cell Environ. 14, 831-841. doi: 10.1111/j.1365-3040.1991.tb0 1446.x

Ito, H., Hiraga, S., Tsugawa, H., Matsui, H., Honma, M., Otsuki, Y., et al. (2000). Xylem-specific expression of wound-inducible rice peroxidase genes in transgenic plants. Plant Sci. 155, 85-100. doi: 10.1016/S0168-9452(00)00209-0

Jefferson, R. (1989). The GUS reporter gene system. Nature 342, 837. doi: 10.1038/ $342837 \mathrm{a} 0$

Jefferson, R. A., Kavanagh, T. A., and Bevan, M. W. (1987). GUS fusions: betaglucuronidase as a sensitive and versatile gene fusion marker in higher plants. EMBO J. 6, 3901.

Kang, H. G., and Singh, K. B. (2000). Characterization of salicylic acid-responsive, Arabidopsis Dof domain proteins: overexpression of OBP3 leads to growth defects. Plant J. 21, 329-339. doi: 10.1046/j.1365-313x.2000.00678.x

Kiyosue, T., Yamaguchi-Shinozaki, K., and Shinozaki, K. (1993). Characterization of two cDNAs (ERD11 and ERD13) for dehydration-inducible genes that encode putative glutathione-S-transferases in Arabidopsis thaliana L. FEBS Lett. 335, 189-192. doi: 10.1016/0014-5793(93)80727-C

Kwak, K. J., Kim, Y. O., and Kang, H. (2005). Characterization of transgenic Arabidopsis plants overexpressing GR-RBP4 under high salinity, dehydration, or cold stress. J. Exp. Bot. 56, 3007-3016. doi: 10.1093/jxb/ eri298

Latha, R., Salekdeh, G. H., Bennett, J., and Swaminathan, M. S. (2004). Molecular analysis of a stress-induced cDNA encoding the translation initiation factor, eIF1, from the salt-tolerant wild relative of rice, Porteresia coarctata. Funct. Plant Biol. 31, 1035-1042. doi: 10.1071/FP03233

Li, A., Li, H., Jin, B., Ye, Q., Zhou, T., Yu, X., et al. (2004). A novel eIF5A complex functions as a regulator of p53 and p53-dependent apoptosis. J. Biol. Chem. 279, 49251-49258. doi: 10.1074/jbc.M407165200

Li, Q., Song, J., Peng, S., Wang, J., Qu, G., Sederoff, R., et al. (2014). Plant biotechnology for lignocellulosic biofuel production. Plant Biotechnol J. 12, 1174-1192. doi: 10.1111/pbi.12273

Liu, L., Liu, Y., Cui, J., Liu, H., Liu, Y. B., Qiao, W. L., et al. (2013). Oxidative stress induces gastric submucosal arteriolar dysfunction in the elderly. World J. Gastroenterol. 19, 9439-9446. doi: 10.3748/wjg.v19.i48.9439
Livak, K. J., and Schmittgen, T. D. (2001). Analysis of relative gene expression data using real-time quantitative PCR and the 2- $\Delta \Delta$ CT Method. Methods 25, 402-408. doi: 10.1006/meth.2001.1262

Ma, J., Li, M., Wang, F., Tang, J., and Xiong, A. S. (2015). Genome-wide analysis of Dof family transcription factors and their responses to abiotic stresses in Chinese cabbage. BMC Genomics 16, 33. doi: 10.1186/s12864-015-1242-9

Minotti, P., Halseth, D., and Sieczka, J. (1994). Field chlorophyll measurements to assess the nitrogen status of potato varieties. HortScience 29, 1497-1500.

Moons, A. (2003). OsGSTU3 and OsGSTU4, encoding tau class glutathione S-transferases, are heavy metal- and hypoxic stress-induced and differentially salt stress-responsive in rice roots. FEBS Lett. 553, 427-432. doi: 10.1016/S00145793(03)01077-9

Passmore, L. A., Schmeing, T. M., Maag, D., Applefield, D. J., Acker, M. G., Algire Mikkel, A., et al. (2007). The eukaryotic translation initiation factors eIF1 and eIF1A induce an open conformation of the 40S ribosome. Mol. Cell 26, 41-50. doi: 10.1016/j.molcel.2007.03.018

Popescu, S. C., Popescu, G. V., Bachan, S., Zhang, Z., Gerstein, M., Snyder, M., et al. (2009). MAPK target networks in Arabidopsis thaliana revealed using functional protein microarrays. Genes Dev. 23, 80-92. doi: 10.1101/gad.1740009

Ptushkina, M., Malys, N., and McCarthy, J. E. (2004). eIF4E isoform 2 in Schizosaccharomyces pombe is a novel stress-response factor. EMBO Rep. 5, 311-316. doi: 10.1038/sj.embor.7400088

Pyronnet, S., and Sonenberg, N. (2001). Cell-cycle-dependent translational control. Curr. Opin. Genet. Dev. 11, 13-18. doi: 10.1016/S0959-437X(00)00150-7

Rangan, L., Rout, A., Sudarshan, M., and Gregorio, G. (2009). Molecular cloning, expression and mapping of the translational initiation factor eIF1 gene in Oryza sativa. Funct. Plant Biol. 36, 442-452. doi: 10.1071/FP08276

Rausell, A., Kanhonou, R., Yenush, L., Serrano, R., and Ros, R. (2003). The translation initiation factor eIF1A is an important determinant in the tolerance to $\mathrm{NaCl}$ stress in yeast and plants. Plant J. 34, 257-267. doi: 10.1046/j.1365313X.2003.01719.x

Saitou, N., and Nei, M. (1987). The neighbor-joining method: a new method for reconstructing phylogenetic trees. Mol. Biol. Evol. 4, 406-425.

Sheen, J. (1996). Ca2+-dependent protein kinases and stress signal transduction in plants. Science 274, 1900-1902. doi: 10.1126/science.274.5294.1900

Shukla, R. K., Raha, S., Tripathi, V., and Chattopadhyay, D. (2006). Expression of CAP2, an APETALA2-family transcription factor from chickpea, enhances growth and tolerance to dehydration and salt stress in transgenic tobacco. Plant Physiol. 142, 113-123. doi: 10.1104/pp.106.081752

Singh, G., Jain, M., Kulshreshtha, R., Khurana, J. P., Kumar, S., and Singh, P. (2007). Expression analysis of genes encoding translation initiation factor 3 subunit g (TaeIF3g) and vesicle-associated membrane protein-associated protein (TaVAP) in drought tolerant and susceptible cultivars of wheat. Plant Sci. 173, 660-669. doi: 10.1016/j.plantsci.2007.09.004

Sun, Y., and Hong, S. (2013). Sensitivity of translation initiation factor eIF1 as a molecular target of salt toxicity to sodic-alkaline stress in the halophytic grass Leymus chinensis. Biochem. Genet. 51, 101-118. doi: 10.1007/s10528-0129546-9

Tanaka, Y., Hibino, T., Hayashi, Y., Tanaka, A., Kishitani, S., Takabe, T., et al. (1999). Salt tolerance of transgenic rice overexpressing yeast mitochondrial Mn-SOD in chloroplasts. Plant Sci. 148, 131-138. doi: 10.1016/S0168-9452(99) 00133-8

Teige, M., Scheikl, E., Eulgem, T., Doczi, R., Ichimura, K., Shinozaki, K., et al. (2004). The MKK2 pathway mediates cold and salt stress signaling in Arabidopsis. Mol. Cell 15, 141-152. doi: 10.1016/j.molcel.2004.06.023

Torres-Schumann, S., Godoy, J. A., and Pintor-Toro, J. A. (1992). A probable lipid transfer protein gene is induced by $\mathrm{NaCl}$ in stems of tomato plants. Plant Mol. Biol. 18, 749-757. doi: 10.1007/BF00020016

Treviño, M. B., and O'Connell, M. A. (1998). Three Drought-Responsive Members of the nonspecific lipid-transfer protein gene family in Lycopersicon pennellii show different developmental patterns of expression. Plant Physiol. 116, 1461-1468. doi: 10.1104/pp.116.4.1461

Vollenweider, S., Weber, H., Stolz, S., Chételat, A., and Farmer, E. E. (2000). Fatty acid ketodienes and fatty acid ketotrienes: michael addition acceptors that accumulate in wounded and diseased Arabidopsis leaves. Plant J. 24, 467-476. doi: 10.1046/j.1365-313x.2000.00897.x

Wang, L., Xu, C., Wang, C., and Wang, Y. (2012). Characterization of a eukaryotic translation initiation factor 5A homolog from Tamarix androssowii involved in 
plant abiotic stress tolerance. BMC Plant Biol. 12:118. doi: 10.1186/1471-222912-118

Wang, Y., Yang, C., and Jiang, J. (2002). The main points and principles of isolating total RNA from ligneous plant tissues [J]. J. Northeast For. Univ. 30, 1-4.

Yang, G., Wang, C., Wang, Y., Guo, Y., Zhao, Y., Yang, C., et al. (2016). Overexpression of ThVHAc1 and its potential upstream regulator, ThWRKY7, improved plant tolerance of Cadmium stress. Sci. Rep. 6:18752. doi: 10.1038/ srep18752

Yang, G., Wang, Y., Xia, D., Gao, C., Wang, C., and Yang, C. (2014). Overexpression of a GST gene (ThGSTZ1) from Tamarix hispida improves drought and salinity tolerance by enhancing the ability to scavenge reactive oxygen species. Plant Cell Tiss. Organ Cult. 117, 99-112. doi: 10.1007/s11240-014-0424-5

Zhang, X., Wang, L., Meng, H., Wen, H., Fan, Y., and Zhao, J. (2011). Maize ABP9 enhances tolerance to multiple stresses in transgenic Arabidopsis by modulating ABA signaling and cellular levels of reactive oxygen species. Plant Mol. Biol. 75, 365-378. doi: 10.1007/s11103-011-9732-x

Zhang, Y., Wang, H., Yang, L., Zhou, X., Zhi, X., Duan, Y., et al. (2016). Egibacter rhizosphaerae gen. nov., sp. nov., an obligately halophilic, facultatively alkaliphilic actinobacterium and proposal of Egibaceraceae fam. nov. and Egibacterales ord. nov. Int. J. Syst. Evol. Microbiol. 66, 283-289. doi: 10.1099/ ijsem.0.000713

Zhang, Y., Wang, Y., and Wang, C. (2012). Gene overexpression and gene silencing in Birch using an Agrobacterium-mediated transient expression system. Mol. Biol. Rep. 39, 5537-5541. doi: 10.1007/s11033-011-1357-2
Zheng, L., Liu, G., Meng, X., Li, Y., and Wang, Y. (2012). A versatile agrobacteriummediated transient gene expression system for herbaceous plants and trees. Biochem. Genet. 50, 761-769. doi: 10.1007/s10528-012-9518-0

Zheng, L., Liu, G., Meng, X., Liu, Y., Ji, X., Li, Y., et al. (2013). A WRKY gene from Tamarix hispida, ThWRKY4, mediates abiotic stress responses by modulating reactive oxygen species and expression of stress-responsive genes. Plant Mol. Biol. 82, 303-320. doi: 10.1007/s11103-013-0063-y

Zhu, C., Li, W., Ma, J., and Ma, X. (2010). Effects of groundwater level on chlorophyll fluorescence characteristics of Tamarix hispida in lower reaches of Tarim River. Ying Yong Sheng Tai Xue Bao 21, 1689-1696.

Zhu, J.-K. (2002). Salt and drought stress signal transduction in plants. Annu. Rev. Plant Biol. 53, 247. doi: 10.1146/annurev.arplant.53.091401. 143329

Conflict of Interest Statement: The authors declare that the research was conducted in the absence of any commercial or financial relationships that could be construed as a potential conflict of interest.

Copyright (c) 2017 Yang, Yu, Wang, Wang and Gao. This is an open-access article distributed under the terms of the Creative Commons Attribution License (CC BY). The use, distribution or reproduction in other forums is permitted, provided the original author(s) or licensor are credited and that the original publication in this journal is cited, in accordance with accepted academic practice. No use, distribution or reproduction is permitted which does not comply with these terms. 